Short communication Annals of Nuclear Medicine 2009, for Kanazawa University Repository

\title{
Attenuation correction of myocardial SPECT by scatter-photopeak window method in normal subjects
}

Koichi Okuda, Kenichi Nakajima, Nobutoku Motomura, Masahiro Kubota, Noriyasu Yamaki, Hisato Maeda, Shinro Matsuo, Seigo Kinuya

Address for correspondence:

Koichi Okuda, Department of Biotracer Medicine, Kanazawa University Graduate School of Medical Science, 13-1 Takara-machi, Kanazawa, 920-8641, Japan;

E-mail: okuda@nmd.m.kanazawa-u.ac.jp

\begin{abstract}
Objective: Segmentation with scatter and photopeak window data using attenuation correction (SSPAC) method can provide a patient-specific non-uniform attenuation coefficient map only by using photopeak and scatter images without X-ray computed tomography (CT). The purpose of this study is to evaluate the performance of attenuation correction (AC) by the SSPAC method on normal myocardial perfusion database.

Methods: A total of 32 sets of exercise-rest myocardial images with Tc-99m-sestamibi were acquired in both photopeak $(140 \mathrm{keV} \pm 10 \%)$ and scatter ( $7 \%$ of lower side of the photopeak window) energy windows. Myocardial perfusion databases by the SSPAC method and non-AC (NC) were created from 15 female and 17 male subjects with low likelihood of cardiac disease using quantitative perfusion SPECT software (QPS). Segmental myocardial counts of a 17-segment model from these databases were compared on the basis of paired $T$ test .

Results: AC average myocardial perfusion count was significantly higher than that in NC in the septal and inferior regions $(\mathrm{P}<0.02)$. On the contrary, AC average count was significantly lower in the anterolateral and apical regions $(\mathrm{P}<0.01)$. Coefficient variation $(\mathrm{CV})$ of the $\mathrm{AC}$ count in the mid, apical and apex regions was lower than that of $\mathrm{NC}$.

Conclusions: The SSPAC method can improve average myocardial perfusion uptake in the septal and inferior regions and provide uniform distribution of myocardial perfusion. The SSPAC method could be a practical method of attenuation correction without X-ray CT.
\end{abstract}

Keywords: Myocardial perfusion imaging, SPECT, non-X-ray based attenuation correction 


\section{Introduction}

Attenuation correction (AC) with $\mathrm{X}$-ray computed tomography (CT) has been sought after for reducing attenuation artifacts [1-8]. The diagnostic accuracy for detection of coronary artery disease will be increased when a myocardial perfusion SPECT (MPS) image is attenuation corrected using patient-specific non-uniform attenuation coefficient maps. Although hybrid SPECT-CT systems play an indispensable role in making non-uniform attenuation coefficient maps, they possess some technical and financial limitations. A potential misregistration between CT and MPS images may occur because of respiratory motion and patient movement [9-10]. The risk of additional radiation exposure by X-ray $\mathrm{CT}$ can not be avoided, and a dedicated SPECT/CT equipment is more expensive than that of conventional SPECT.

To overcome these problems, a novel AC technique called "segmentation of scatter and photopeak window data for attenuation correction (SSPAC)" method has been developed [11-12]. This new technique can make a non-uniform attenuation coefficient map, which uses only photopeak and scatter-window images obtained from SPECT acquisition. The SSPAC method may allow for correction of attenuation artifacts without X-ray CT thus avoiding the risk of additional radiation exposure.

In the present study, attenuation corrected gender-specific databases were generated to assess the effects of correction myocardial perfusion. The purpose of this study was to evaluate the standard perfusion patterns by this new approach for attenuation correction.

\section{Materials and methods}

\section{Study population}

A total of 32 subjects (17 males and 15

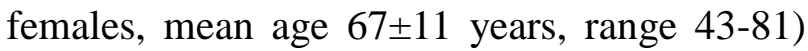
with low likelihood of cardiac disease were selected consecutively, and gated MPS was performed from August 10, 2006 to December 20, 2006. These subjects underwent exercise $(n=10)$ or pharmacological $(n=22)$ stress and rest studies. Indications for MPI included electrocardiographic abnormalities to rule out ischemia $(n=11)$, screening for ischemia $(n=6)$ and preoperative risk stratification $(n=15)$. The possibility of ischemic heart disease was denied out by subsequent clinical studies in all patients.

\section{Attenuation correction with SSPAC method}

Patient specific AC maps were generated by the SSPAC method, which was developed at Toshiba Medical Systems, Tokyo, Japan. The SSPAC method only requires dual or triple energy window acquisition: emission by $140 \mathrm{keV} \pm 10 \%$, (2) scatter by $7 \%$ of the lower side of the emission window (3) septal penetration by $7 \%$ of the higher side of the emission window. The procedure for $\mathrm{AC}$ method was as follows: First the contour maps of the body, lung, heart and liver outlines were generated from photopeak and scatter images. The body outlines were segmented from differential filtered scatter images to calculate the distance from the center of the body for each radial angle. The lung contours were determined by elliptical approximation using filtered scatter images. Segmentation of the myocardium and liver was obtained from photopeak images. The average models of 
mediastinum and thoracic spine were obtained by X-ray $\mathrm{CT}$ and applied to generate an $\mathrm{AC}$ map. All contour maps were combined and segmented by three regions of bone, soft tissue and lung. An attenuation coefficient map was generated to apply attenuation coefficient values of bone, soft tissue and lung to attenuation coefficients of $0.280 / \mathrm{cm}, 0.150 / \mathrm{cm}$, and $0.053 / \mathrm{cm}$ respectively. Finally, a Gaussian filter was applied to an attenuation coefficient map for the system resolution compensation.

\section{Image acquisition}

Stress and rest gated myocardial perfusion studies were performed during a 1-day protocol using $300 \mathrm{MBq}$ and $740 \mathrm{MBq}$ ${ }^{99 \mathrm{~m}}$ Tc-sestamibi respectively. MPS images were acquired by using triple-detector scintillation cameras (9300A, Toshiba Medical Systems, Tokyo, Japan) with low-energy high-resolution collimators. A total of 60 projections were obtained from a $360^{\circ}$ circular orbit with $64 \times 64$ matrix. The pixel size was $6.4 \mathrm{~mm}$. Data from emission and scatter photopeaks were collected. For gated acquisition, the division of the RR interval was 16 frames.

\section{Data processing and analysis}

The MPS reconstruction was performed with a GMS-7700R workstation (Toshiba Medical Systems) for both AC and non-attenuation correction (NC) images. Scatter correction was performed using scatter data obtained from the lower side of the main photopeak window. The AC images were reconstructed by an ordered subsets-expectation maximization algorithm with 4 iterations and 10 subsets using the attenuation coefficient map. The NC images were also reconstructed using the same condition. Reconstructed short-axis images for $\mathrm{AC}$ and $\mathrm{NC}$ were analyzed with a quantitative perfusion SPECT and gated SPECT software (QPS/QGS, Cedars-Sinai Medical Center, Los Angeles, CA, USA). A QPS software algorithm was used to generate perfusion polar maps of 17 segments. $\mathrm{AC}$ and $\mathrm{NC}$ databases were calculated on the basis of the QPS software for both genders. Ejection fraction (EF), end-diastolic volume (EDV) and end-systolic volume (EDV) were obtained form QGS analysis.

\section{Statistical analysis}

Segmental values of the databases were expressed as the average and standard deviation, which were calculated by QPS software. A paired $t$ test was used to compare differences in the databases for $\mathrm{AC}$ and $\mathrm{NC}$. Since CV values were calculated to compare the uniformity of perfusion between $\mathrm{AC}$ and $\mathrm{NC}$, differences were also assessed by a paired t test. All statistical tests were two-tailed, and a $\mathrm{P}$ value of less than 0.05 was considered to be significant.

\section{Results}

\section{Left ventricular function}

EF, EDV and ESV values derived from normal subjects were $70.9 \pm 7.7 \%, 62.1 \pm 13.7 \mathrm{ml}$, $18.8 \pm 8.3 \mathrm{ml}$ for females and $64.9 \pm 9.1 \%$, $81.8 \pm 17.8 \mathrm{ml}, \quad 29.1 \pm 9.9 \mathrm{ml}$ for males, respectively $(\mathrm{p}=0.056,0.002$ and 0.004$)$.

\section{Statistical differences in database analysis}

Averaged perfusion polar maps are shown in Figure 1, and calculated segmental 
average and standard deviation values in 17-segment models for stress, and rest conditions are shown in Table 1. The attenuation corrected polar maps demonstrated homogeneous count distribution when the anterior and inferior counts were compared (Figure 1). Especially in the mid anteroseptal, inferoseptal and inferior segments, attenuation corrected myocardial perfusion counts were significantly higher than that of the NC ( $\mathrm{p}=<0.0001,0.0002$ and 0.0003 , respectively). Although the whole regions of myocardial were corrected by the SSPAC method, the segmental average value in the mid anterolateral segment showed a lower value using AC in comparison to NC ( $<<0.0001)$. Furthermore, apical thinning at the apex segment was demonstrated after AC, and the attenuation corrected average value in the apex showed a lower value $(\mathrm{p} \leq 0.0128)$. Paired $t$ test results for females versus males and AC versus NC are shown in Table 2. Regarding gender differences, the mid and apical inferior regions showed lower $\mathrm{p}$ values with both AC and NC. Nevertheless, segmental $\mathrm{p}$ values differed significantly in the mid inferoseptal during rest condition and mid inferolateral during stress condition. When comparing $\mathrm{AC}$ and $\mathrm{NC}$, the mid-anteroseptal, inferoseptal inferior and anterolateral segments showed significanty higher values in all cases.

\section{Coefficient of variation values analysis}

Calculated CV values in the mid, apical and apex regions are shown in Figure 2. Attenuation corrected $\mathrm{CV}$ values showed a lower variation in both genders; the mean \pm SD values for females with $\mathrm{AC}$ and $\mathrm{NC}$ for stress and rest were $6.1 \pm 1.1 \%, 5.8 \pm 1.5 \%, 7.0 \pm 2.4 \%$ and $7.2 \pm 2.6 \%$, respectively, and for males, these values were $7.2 \pm 1.7 \%$, $6.8 \pm 1.3 \%, 9.3 \pm 1.7 \%$ and $9.8 \pm 1.4 \%$ respectively. There were statistically significant differences between $\mathrm{AC}$ and $\mathrm{NC}$ values in males $(\mathrm{p}=0.04)$.

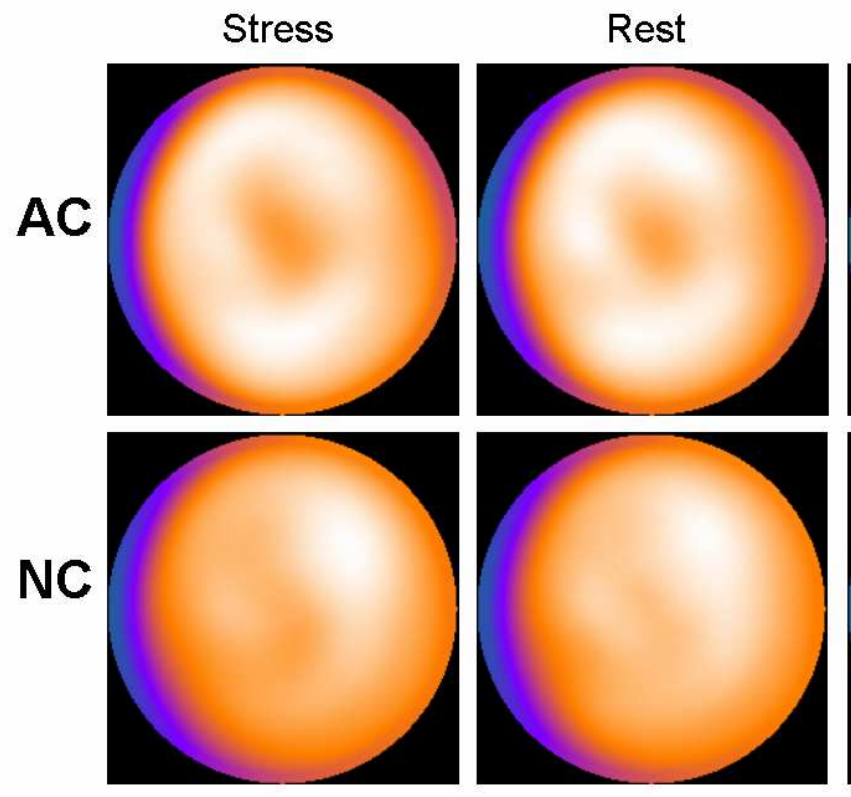

Female

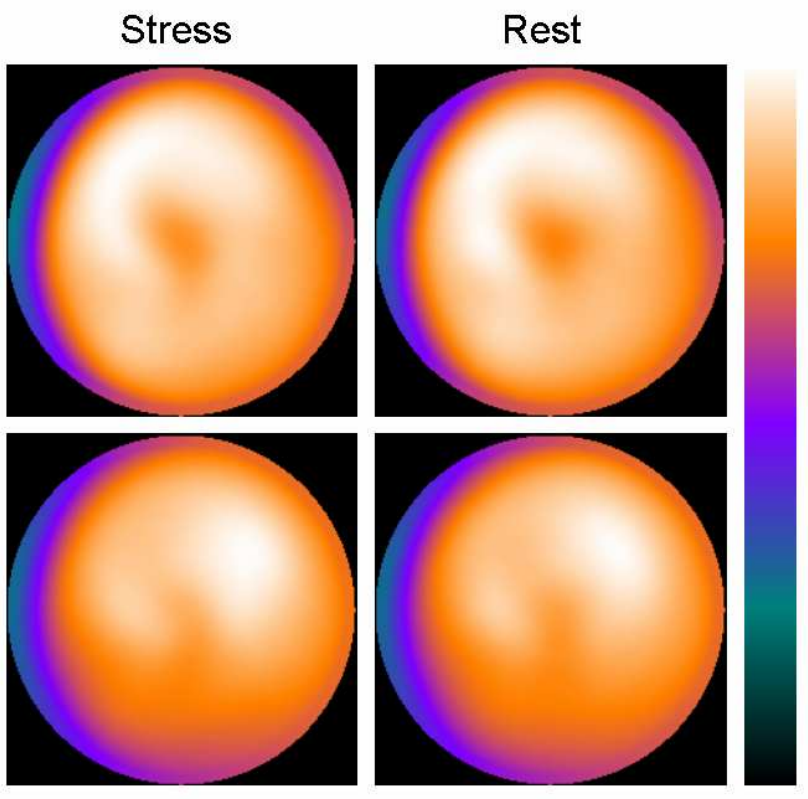

Male

Fig. 1 Comparison of the average polar maps for AC and NC in both genders. The differences in the septal and inferior counts between $\mathrm{AC}$ and $\mathrm{NC}$ are shown. 
Table 1 Segmental average and standard deviation values in the polar maps with AC and NC

\begin{tabular}{|c|c|c|c|c|c|c|c|c|}
\hline & \multicolumn{4}{|l|}{$A C$} & \multicolumn{4}{|l|}{$\mathrm{NC}$} \\
\hline & \multicolumn{2}{|l|}{ Stress } & \multicolumn{2}{|l|}{ Rest } & \multicolumn{2}{|l|}{ Stress } & \multicolumn{2}{|l|}{ Rest } \\
\hline & Female & Male & Female & Male & Female & Male & Female & Male \\
\hline Basal-A & $66 \pm 7$ & $66 \pm 7$ & $73 \pm 5$ & $67 \pm 7$ & $70 \pm 7$ & $65 \pm 6$ & $72 \pm 5$ & $65 \pm 6$ \\
\hline Basal-AS & $57 \pm 8$ & $56 \pm 9$ & $62 \pm 8$ & $56 \pm 9$ & $57 \pm 8$ & $53 \pm 8$ & $59 \pm 6$ & $53 \pm 8$ \\
\hline Basal-IS & $62 \pm 7$ & $64 \pm 9$ & $65 \pm 8$ & $63 \pm 7$ & $58 \pm 7$ & $54 \pm 7$ & $57 \pm 8$ & $54 \pm 5$ \\
\hline Basal-I & $71 \pm 7$ & $68 \pm 7$ & $75 \pm 6$ & $68 \pm 6$ & $67 \pm 7$ & $59 \pm 5$ & $66 \pm 7$ & $59 \pm 5$ \\
\hline Basal-IL & $62 \pm 4$ & $61 \pm 5$ & $66 \pm 5$ & $62 \pm 6$ & $68 \pm 3$ & $64 \pm 5$ & $68 \pm 5$ & $64 \pm 5$ \\
\hline Basal-AL & $58 \pm 3$ & $58 \pm 6$ & $62 \pm 4$ & $59 \pm 6$ & $70 \pm 4$ & $66 \pm 5$ & $71 \pm 3$ & $66 \pm 5$ \\
\hline Mid-A & $91 \pm 5$ & $92 \pm 4$ & $93 \pm 4$ & $92 \pm 5$ & $89 \pm 5$ & $88 \pm 5$ & $89 \pm 3$ & $86 \pm 5$ \\
\hline Mid-AS & $86 \pm 6$ & $88 \pm 6$ & $89 \pm 4$ & $88 \pm 5$ & $81 \pm 4$ & $79 \pm 5$ & $81 \pm 4$ & $79 \pm 5$ \\
\hline Mid-IS & $83 \pm 5$ & $84 \pm 5$ & $87 \pm 5$ & $83 \pm 4$ & $76 \pm 6$ & $72 \pm 5$ & $75 \pm 7$ & $71 \pm 4$ \\
\hline Mid-I & $90 \pm 5$ & $83 \pm 7$ & $93 \pm 4$ & $83 \pm 6$ & $83 \pm 7$ & $73 \pm 4$ & $82 \pm 7$ & $71 \pm 4$ \\
\hline Mid-IL & $83 \pm 5$ & $80 \pm 6$ & $87 \pm 5$ & $81 \pm 5$ & $87 \pm 3$ & $82 \pm 4$ & $87 \pm 4$ & $81 \pm 3$ \\
\hline Mid-AL & $83 \pm 4$ & $84 \pm 6$ & $87 \pm 5$ & $85 \pm 5$ & $93 \pm 3$ & $93 \pm 3$ & $95 \pm 1$ & $92 \pm 3$ \\
\hline Apical-A & $87 \pm 5$ & $87 \pm 3$ & $87 \pm 4$ & $89 \pm 4$ & $90 \pm 3$ & $89 \pm 4$ & $89 \pm 3$ & $90 \pm 5$ \\
\hline Apical-S & $88 \pm 4$ & $90 \pm 4$ & $89 \pm 3$ & $89 \pm 4$ & $87 \pm 4$ & $86 \pm 5$ & $85 \pm 5$ & $85 \pm 4$ \\
\hline Apical-I & $87 \pm 4$ & $83 \pm 4$ & $88 \pm 4$ & $83 \pm 4$ & $85 \pm 4$ & $78 \pm 4$ & $82 \pm 6$ & $77 \pm 4$ \\
\hline Apical-L & $85 \pm 4$ & $82 \pm 3$ & $86 \pm 5$ & $85 \pm 3$ & $92 \pm 3$ & $89 \pm 3$ & $91 \pm 4$ & $90 \pm 4$ \\
\hline Apex & $80 \pm 5$ & $77 \pm 6$ & $80 \pm 5$ & $79 \pm 5$ & $87 \pm 3$ & $83 \pm 4$ & $85 \pm 5$ & $84 \pm 5$ \\
\hline
\end{tabular}

A:anterior,AS:anteroseptal,IS:inferoseptal,I:inferior,IL:inferolateral,AL:anterolateral,A:Antorior

Table 2 Comparison of gender differences in $\mathrm{AC}$ and NC, and attenuation differences.

\begin{tabular}{|c|c|c|c|c|c|c|c|c|}
\hline & \multicolumn{4}{|c|}{$P$ values for Female versus Male } & \multicolumn{4}{|c|}{$P$ values for $A C$ versus $N C$} \\
\hline & \multicolumn{2}{|l|}{$A C$} & \multicolumn{2}{|l|}{ NC } & \multicolumn{2}{|l|}{ Stress } & \multicolumn{2}{|l|}{ Rest } \\
\hline & Stress & Rest & Stress & Rest & Female & Male & Female & Male \\
\hline Basal-A & ns & 0.0107 & ns & 0.0051 & ns & ns & ns & 0.0038 \\
\hline Basal-AS & ns & 0.0471 & ns & ns & ns & 0.0145 & 0.0227 & 0.0028 \\
\hline Basal-IS & ns & ns & ns & ns & 0.0052 & $<0.0001$ & $<0.0001$ & $<0.0001$ \\
\hline Basal-I & ns & 0.0037 & 0.0108 & 0.0012 & 0.0023 & $<0.0001$ & $<0.0001$ & $<0.0001$ \\
\hline Basal-IL & ns & ns & 0.0450 & 0.0257 & $<0.0001$ & 0.0083 & ns & ns \\
\hline Basal-AL & ns & ns & ns & 0.0080 & $<0.0001$ & $<0.0001$ & $<0.0001$ & $<0.0001$ \\
\hline Mid-A & ns & ns & ns & ns & ns & 0.0023 & 0.0082 & $<0.0001$ \\
\hline Mid-AS & ns & ns & ns & ns & $<0.0001$ & $<0.0001$ & $<0.0001$ & $<0.0001$ \\
\hline Mid-IS & ns & ns & ns & 0.0281 & 0.0002 & $<0.0001$ & $<0.0001$ & $<0.0001$ \\
\hline Mid-I & 0.0147 & $<0.0001$ & 0.0001 & $<0.0001$ & 0.0003 & $<0.0001$ & $<0.0001$ & $<0.0001$ \\
\hline Mid-IL & ns & 0.0022 & 0.0050 & 0.0020 & 0.0031 & ns & ns & ns \\
\hline Mid-AL & ns & ns & ns & ns & $<0.0001$ & $<0.0001$ & $<0.0001$ & $<0.0001$ \\
\hline Apical-A & ns & ns & ns & ns & 0.0120 & ns & ns & ns \\
\hline Apical-S & ns & ns & ns & ns & ns & 0.0082 & 0.0294 & 0.0005 \\
\hline Apical-I & 0.0353 & 0.0018 & 0.0005 & 0.0007 & ns & 0.0058 & 0.0019 & $<0.0001$ \\
\hline Apical-L & ns & ns & ns & ns & 0.0014 & $<0.0001$ & 0.0037 & $<0.0001$ \\
\hline Apex & ns & ns & ns & ns & 0.0005 & 0.0024 & 0.0128 & 0.0012 \\
\hline
\end{tabular}




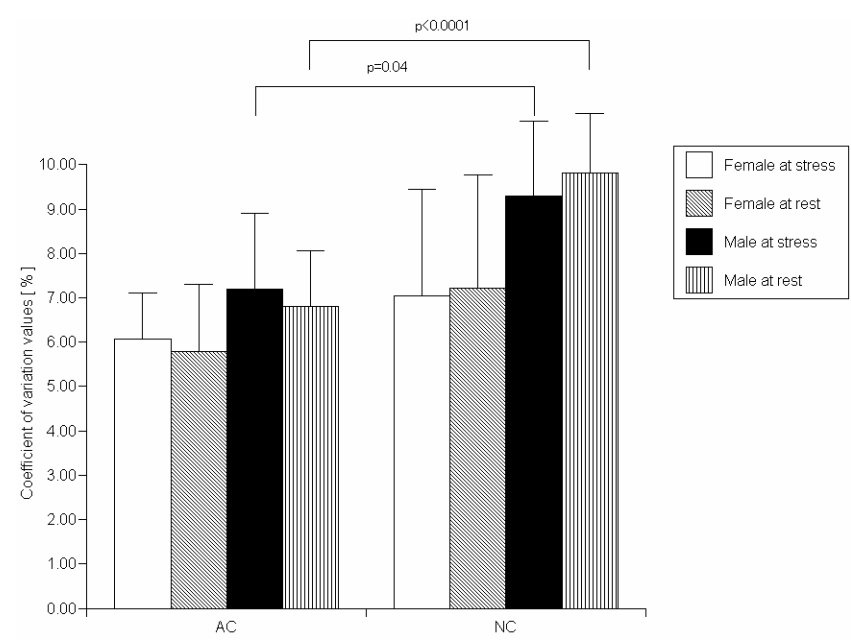

Fig. 2 The coefficient of variation (CV) values in the mid, apical and apex regions

\section{Discussion}

The SSPAC method could reduce attenuation artifacts and changed calculated segmental average values of myocardial counts in comparison with NC databases. Attenuation corrected myocardial perfusion counts were more homogeneous than NC images and the anteroseptal, inferoseptal and inferior counts were increased. Moreover, attenuation corrected databases should be created for quantitative analysis of $\mathrm{AC}$ images when the SSPAC method is applied to clinical uses.

MPS images frequently showed attenuation artifacts in males and females due to attenuation from diaphragm and beast. There were non-attenuation-corrected normal databases for the Japanese population in which the polar maps showed a lower count distribution in the inferior region in males. Most of the female subjects did not show a significant lower count in the anterior segment. Nakajima et al. reported that differences in sex were significant in the mid- and apical inferior segments, showing lower values in men.
Moreover, there were no significant differences in the anterior segments between genders [13]. This finding shows that the body habitus is more homogeneous in eastern Asian females and males than in European and American populations. In attenuation corrected databases, attenuation artifacts in the inferior segment were corrected in males, and the count distribution in each polar map was more homogeneous in both genders. An X-ray based AC study report also showed that attenuation corrected polar maps increased global uniformity of the count distribution [4].

A major problem is that attenuation corrected count distribution in the apex region sometimes shows a moderate defect. This apical thinning is a well known phenomenon when using the X-ray based AC method [14, 15]. This phenomenon may induce false-positive perfusion defects and may reduce the specificity in a normal perfusion. In the case of the SSPAC method, similar phenomenons have also occurred. Our previous studies have demonstrated that attenuation coefficient maps obtained from SSPAC method and transmission CT showed the similar segmentation of heart, liver, and torso [11, 12]. Therefore, if co-registarion with attenuation coefficient maps and MPS images were correctly performed, similar results might be anticipated by both SSPAC and X ray CT based AC methods.

Some reports evaluated the apical thinning in phantom and clinical studies by AC $[8,14]$. In the phantom study, phantom sizes, breast tissues and reconstruction parameters were thought to be responsible for apical thinning, whereas apical thinning was a consistent finding independent on gender or 
body habitus in the clinical study. Therefore, variability in the apical segment may be affected by increasing counts in the inferoseptal regions in comparison with the apical region in addition to apical wall motion in clinical study.

Considering that the count distributions differed between $\mathrm{AC}$ and $\mathrm{NC}$ databases, AC-specific database should be created in clinical practice. Count distribution between $\mathrm{AC}$ and $\mathrm{NC}$ differed between stress and rest conditions for both gender. A X-ray CT based AC study showed that gender differences were not statistically significant after AC [4]. Although quantitative analysis of myocardial perfusion required gender-specific databases, single attenuation corrected databases independent on genders would be convenient.

This study has several limitations. CT image resolution is better than that of MPS image for generating an attenuation coefficient map. Since the body contours were obtained from differential filtered scatter image, information of breast tissues was not precisely traced. The effect of dense breasts in the anterior wall should be evaluated in further clinical studies. Thus, improvement of the algorithm for detecting a body contour of breast would be required. However, Wolak et al. reported that there are no significant diagnostic differences with and without AC in women [16]. Since the diagnostic difference between SSPAC and SPECT-CT methods has not been examined, further investigation is required to evaluate the diagnostic efficacy in large numbers of patients with coronary artery disease.
Attenuation corrected myocardial perfusion SPECT images could be obtained without X-ray CT data. Significant segmental differences were observed in some regions between AC and NC polar maps. Since myocardial perfusion became homogeneous in the mid, apical and apex regions in the normal subjects study, attenuation corrected normal database should be generated for clinical practice. Further studies in patients with coronary artery disease should be indicated to evaluate the clinical efficacy of the SSPAC method.

\section{Acknowledgement}

This work was supported partly by Grants-in-Aid for Scientific Research in Japan (No.19591403)

\section{References}

1. Heller GV, Links J, Bateman TM, Ziffer JA, Ficaro E, Cohen MC, et al. American Society of Nuclear Cardiology and Society of Nuclear Medicine joint position statement: attenuation correction of myocardial perfusion SPECT scintigraphy. J Nucl Cardiol. 2004; 11: 229-30.

2. Hendel RC, Berman DS, Cullom SJ, Follansbee W, Heller GV, Kiat H, et al. Multicenter clinical trial to evaluate the efficacy of correction for photon attenuation and scatter in SPECT myocardial perfusion imaging. Circulation. 1999; 99: 2742-49.

3. Patton JA, Turkington TG. SPECT/CT physical principles and attenuation correction. J Nucl Med Technol. 2008; 36: 1-10.

4. Grossman GB, Garcia EV, Bateman TM, Heller GV, Johnson LL, Folks RD, et al. Quantitative 
Tc-99m sestamibi attenuation-corrected SPECT: development and multicenter trial validation of myocardial perfusion stress gender-independent normal database in an obese population. J Nucl Cardiol. 2004; 11: 263-72.

5. Thompson RC, Heller GV, Johnson LL, Case JA, Cullom SJ, Garcia EV, et al. Value of attenuation correction on ECG-gated SPECT myocardial perfusion imaging related to body mass index. J Nucl Cardiol. 2005; 12: 195-202.

6. Utsunomiya D, Tomiguchi S, Shiraishi S, Yamada K, Honda T, Kawanaka K, et al. Initial experience with X-ray CT based attenuation correction in myocardial perfusion SPECT imaging using a combined SPECT/CT system. Ann Nucl Med. 2005; 19: 485-9.

7. Apostolopoulos DJ, Spyridonidis T, Skouras T, Giannakenas C, Savvopoulos C, Vassilakos PJ. Comparison between 180 degrees and 360 degrees acquisition arcs with and without correction by CT-based attenuation maps in normal hearts at rest. Nucl Med Commun. 2008; 29: 110-9.

8. Ficaro EP, Fessler JA, Shreve PD, Kritzman JN, Rose PA, Corbett JR. Simultaneous transmission/emission myocardial perfusion tomography. Diagnostic accuracy of attenuation-corrected 99m-Tc-sestamibi single-photon emission computed tomography. Circulation. 1996; 93: 463-73.

9. Goetze S, Brown TL, Lavely WC, Zhang Z, Bengel FM. Attenuation correction in myocardial perfusion SPECT/CT: effects of misregistration and value of reregistration. J Nucl Med. 2007; 48:
1090-95.

10. Fricke H, Fricke E, Weise R, Kammeier A, Lindner $\mathrm{O}$, Burchert W. A method to remove artifacts in attenuation-corrected myocardial perfusion SPECT Introduced by misalignment between emission scan and CT-derived attenuation maps. J Nucl Med. 2004; 45: 1619-25

11. Maeda H, Natsume T, Yamaki N, Shirakawa S, Kikukawa K, Toyama $\mathrm{H}$, et al. Attenuation correction for myocardial SPECT: segmentation with scatter and photopeak window data for attenuation correction (SSPAC) method. J Nucl Med. 2002; 43(Supplement): 230P

12. Shirakawa S, Yamaki N, Natsume T, Hasebe S, Toyama H, Maeda K, et al. A computer simulation method for attenuation correction in myocardial SPECT. J Nucl Med. 2003; 44(Supplement): 275P

13. Nakajima K, Kumita S, Ishida $Y$, Momose $M$, Hashimoto J, Morita K, et al. Creation and characterization of Japanese standards for myocardial perfusion SPECT: database from the Japanese Society of Nuclear Medicine Working Group. Ann Nucl Med. 2007; 21: 505-11

14. Purser NJ, Armstrong IS, Williams HA, Tonge CM, Lawson RS. Apical thinning: real or artefact? Nucl Med Commun. 2008; 29: 382-89.

15. Links JM, Becker LC, Anstett F. Clinical significance of apical thinning after attenuation correction. J Nucl Cardiol. 2004; 11: 26-31.

16. Wolak A, Slomka PJ, Fish MB, Lorenzo S, Berman DS, Germano G. Quantitative diagnostic performance of myocardial perfusion SPECT with attenuation correction in women. J Nucl Med. 2008; 49: 915-22. 